Methods: In this randomized-controlled study, one recent onset psychosis $(\mathrm{ROP})$ patient arm $(\mathrm{n}=18)$ underwent a 6-week (10-hour) computerized SCT (Brain HQ, Posit Science, https://www.brainhq.com/), while another naturalistic arm $(n=18)$ received treatment as usual (TAU). Both treatment arms were assessed on a battery of neurocognitive tests and underwent a multimodal imaging protocol, including a 10 min restingstate fMRI, at two timepoints (baseline, T0; follow-up, FU). Seed-based voxel-wise rsFC was performed and individual-level rsFC correlation maps were calculated between the bilateral medial prefrontal cortex (mPFC) and the whole brain.

Results: The SCT group showed significant improvements in the domain of spatial working memory $(\mathrm{p}<0.05)$, processing speed $(\mathrm{p}<0.05)$ and resilience to both immediate and delayed memory decline over 6 weeks $(\mathrm{p}<0.05)$, as compared to TAU. Comparison of FC between the two measurement time points, suggested increased FC between $\mathrm{MPFC}$ and left inferior temporal gyrus (ITG), as well as increased FC between mPFC and left somatosensory area in ROP patients that underwent SCT relative to TAU.

Discussion: We have shown improvements in processing speed, verbal memory, and spatial working memory that agree with previous studies using computerized cognitive interventions. Moreover, the improvement in the spatial working memory domain was significant in the most demanding test condition with 10 elements - indicative of benefits from the fine-tuning of higher-level executive functions. The neuroimaging results also suggested that the improvements may have been mediated by the improvement of FC in regions typically associated with social cognition and facial recognition (Adolphs et al., 2009). These results are in line with recent studies investigating not only the feasibility of SCT as an intervention, but also the effects on cognition and underlying neural alterations resulting from intensive computerized neurocognitive interventions (Hooker et al., 2012; Nahum et al., 2014; Subramaniam et al., 2014). Future studies using machine learning methods will be necessary to determine functional biomarkers in order to personalize SCT at the individual-level.

\section{F71. THE STRUCTURE OF NEUROCOGNITION ACROSS CHILDHOOD AND ADULTHOOD IN YOUNG PEOPLE WITH PSYCHOSIS.}

\author{
Josephine Mollon*, ${ }^{*}$, Anthony David ${ }^{2}$, Stanley Zammit ${ }^{3}$, \\ Glyn Lewis ${ }^{4}$, Avi Reichenberg ${ }^{5}$ \\ ${ }^{1}$ Yale University; ${ }^{2}$ Institute of Psychiatry, King's College London; \\ ${ }^{3}$ Cardiff University \& University of Bristol; ${ }^{4}$ University College \\ London; ${ }^{5}$ Icahn School of Medicine at Mount Sinai
}

Background: There is substantial evidence for connection abnormalities in the brains of schizophrenia patients. However, little is known about the structure of cognitive functioning across the psychosis spectrum.

Methods: We used data from the Avon Longitudinal Study of Parents and Children (ALSPAC). Data from all individuals who underwent cognitive testing at age 8 and psychiatric assessment at 18 years were used to examine network structure of cognition in childhood (age 8). A subsample of individuals who underwent further cognitive testing at age 20 was used to examine change in cognitive network structure between childhood (age 8) and adulthood (age 20). Networks comprised nodes (cognitive tests) joined together by edges (partial correlations). Organization of subnetworks by cognitive domains (verbal, perceptual, working memory and processing speed) and measures indicating 1) important cognitive tests or hubs, 2) network integration and 3) network density, were examined. Participants with non-affective psychotic disorder, affective psychotic disorder, psychotic experiences and depression were compared to controls.

Results: In childhood, affective and non-affective psychosis groups showed disruption to cognitive subnetworks and hubs, as well as greater network connectivity $(\beta=0.44, p<.001, \beta=0.16, p<.001)$, dysconnectivity $(\beta=-0.47$, $\mathrm{p}<.001, \quad \beta=-0.19, \mathrm{p}=.002)$, integration $(\beta=-12.7, \mathrm{p}<.001, \quad \beta=-10.2$, $\mathrm{p}<.001)$ and density $(\beta=0.49, \mathrm{p}<.001, \beta=0.17, \mathrm{p}<.001)$. The psychotic experiences group showed intact subnetworks and hubs, but increased network integration $(\beta=-5.5, \mathrm{p}<.001)$ and density $(\beta=0.02, \mathrm{p}=.04)$. The depression group also showed intact subnetworks and hubs, but increased integration $(\beta=-5.9, p<.001)$. Between childhood and adulthood increasing density was seen in the psychotic experiences group $(\beta=0.09, p=.04)$, and the depression group showed increasing integration $(\beta=-3.15, \mathrm{p}=.04)$. Controls showed increasing reliance on the working memory hub between childhood and adulthood, while all other groups remained reliant on attention and visuospatial abilities.

Discussion: Overall, individuals with psychotic disorder showed substantial qualitative and quantitative differences in cognitive network structure. Individuals with psychotic experiences and depression showed more subtle deviations. Abnormalities in cognitive network structure were seen even in the absence of cognitive impairment, suggesting the importance of looking beyond deficits to how performance is achieved.

\section{F72. NEUROCOGNITION AND ADAPTIVE FUNCTIONING IN THE 22Q11.2 DELETION SYNDROME MODEL OF SCHIZOPHRENIA}

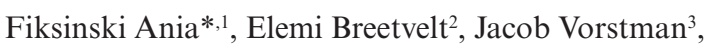
Eva Chow ${ }^{2}$, Erin Lee ${ }^{2}$, Lisa Palmer ${ }^{2}$, Erik Boot ${ }^{2}$, Nancy Butcher ${ }^{2}$, Rene Kahn ${ }^{1}$, Anne Bassett ${ }^{2}$

${ }^{1}$ UMC Utrecht; ${ }^{2}$ University of Toronto; ${ }^{3}$ UMC Utrecht, University of Toronto

Background: Identifying factors that influence functional outcome is an important goal in schizophrenia research. These factors, including overall cognitive functioning (IQ) and more specific domains of neurocognitive functioning, may not only aid in identifying those individuals at greatest risk for poor functional outcome but could inform potentially targetable treatment objectives. The 22q11.2 deletion syndrome (22q11DS) is a unique genetic model with high risk (20-25\%) for schizophrenia. This study aimed to identify potentially targetable domains of neurocognitive functioning associated with functional outcome in adults with 22q11DS.

Methods: Using data available from a comprehensive battery of 15 neurocognitive tests for 99 adults with 22q11DS ( $n=43$ with schizophrenia) we derived four domains of neurocognition (Verbal memory, Visual memory, Motor functioning, and Executive performance) using a principal component analysis. To investigate the association of these domains with adaptive functioning, we used Vineland Adaptive Behavior Scales (VABS) data available for 84 subjects in a logistic regression model that accounted for the effects of schizophrenia status and overall intellectual level.

Results: The regression model explained $46.8 \%$ of the variance in overall functional outcome $(\mathrm{p}<0.0001)$ and $47.7 \%$ of the variance on the daily living skills subdomain $(\mathrm{p}<0.0001)$. Executive performance was significantly associated with subsequent functional outcome ( $\mathrm{p}=0.046)$; age and schizophrenia were also significant factors. VABS adaptive functioning scale scores were higher in those with better performance on Executive domain tests, no psychotic illness, and older age. The effects of Executive Performance on functioning did not significantly differ between those with and without psychotic illness.

Discussion: The significant relationship between Executive Performance and functional outcome is a novel addition to our understanding of cognitive factors that may contribute to the variability in functional outcome in schizophrenia high-risk groups. The results provide impetus for further studies of Executive Performance as a potential target of early intervention strategies to mitigate risk for schizophrenia and functional deterioration. 